\title{
Development of Seamless Rolling Machine for Solar Greenhouse Yongwei Yuan ${ }^{1, a}$, Degang Kong ${ }^{1, b}$, Shanshan $\mathrm{Li}^{1, \mathrm{c}}$, Jinggang $\mathrm{Yi}^{1, \mathrm{~d}}$, Jiangtao $\operatorname{Liu}^{1, \mathrm{e}}$
}

${ }^{1}$ Mechanical and Electrical Engineering College Agricultural University of Hebei, Baoding 071001, China.

ayyw0314@126.com, b870714576@qq.com, chdlishanshan@126.com, ,jdyjg@hebau.edu.cn, eliujiangtao2003@126.com

Keywords: Solar greenhouse, Rolling machine, Seamless device, Remote control

\begin{abstract}
The rolling machine has been widely used in agricultural facilities,For the pre-push rolling machine has about two-metre-wide blanket below the reduction gear can not be automatic rolled up and down problems, Designed a seamless device which can automatic roll up and down the intermediate blanket. The device has a folding function, Runing-time, It is open $150^{\circ}$ to avoid collision with the surface of the greenhouses. After contacted with the ground, it is folding. The control system has site manual, short-range control and SMS remote control three modes, Using location switch and time protection to achieve double protection. The test result shows that the machine is not only running well but also safe and convenient to operate.
\end{abstract}

\section{Introduction}

Solar greenhouse, Not only shorten the crop growth cycle, provide anti-season vegetables, but also can greatly improve the survival rate of crops. In the background of mechanization of agricultural facilities and the development of intelligent, Rolling machine as a small agricultural machinery has been widely used ${ }^{[1]}$.

Rolling machine appeared greatly promoted the development of mechanization in Greenhouse, While, reduced the labor intensity of the farmer ${ }^{[2]}$. Most rolling machine installed in the middle position, Through the rolling axis connected on both sides of the reduction gear to realizes the both sides of blankets automatically rolling up and down, But there are about two-metre-wide blanket below the reduction gear can not be automatic rolled up and down, As shown in figure 1.

Currently rolling machines generally use the manual control, Not only there is a big security risk, but also a waste of time. It is an urgent need to develop a seamless rolling machine, achieving intermediate blankt automatic up and down, And can be achieved manually, short-range control and remote control of mobile phone messages.

\section{Seamless rolling machine overall design}

\subsection{Overall structure}

Solar greenhouse seamless rolling machines general structure diagram shown in Figure 2, It mainly consists of power mechanism,rolling axis, support mechanism, Seamless device,limit protection device and other components. Power mechanism is composed of three-phase induction motor and a worm reduction gear. Both sides of the gear output shaft are connected with rolling axis, A sprocket is mounted on the right side of the gear output shaft and as a power device for seamless device. the device has two clamping sleeves connected with the rolling axis, At the top of the clamping sleeve, it has two hard limits, so that the device has a folding resistance. 


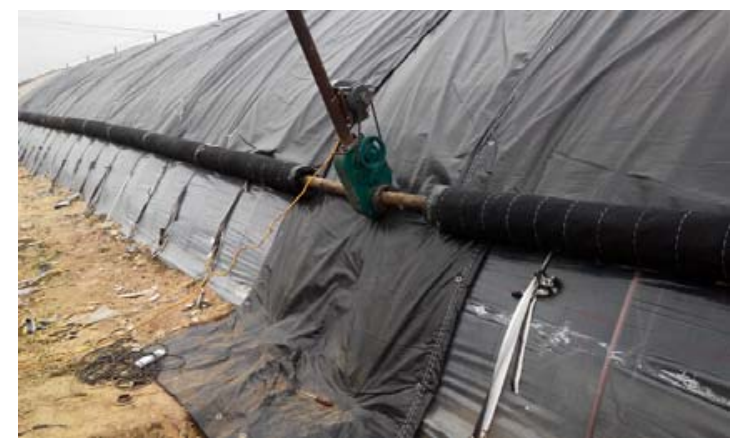

Figure 1 Traditional solar greenhouse rolling machines work results

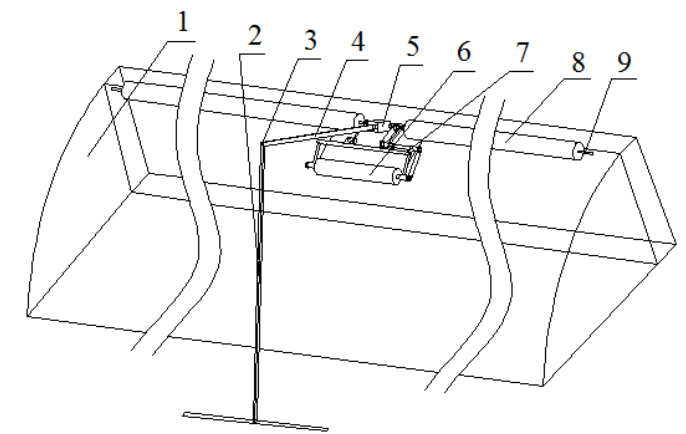

1 Solar greenhouse 2 Support arm 3Hinge 4 Rocker arm 5 Power mechanism 6 Intermediate blanket 7 Seamless device 8 Blankets 9 Rolling axis

Figure 2 Solar greenhouse seamless rolling machines overall structure diagram

\subsection{Working principle}

In the course of working, The motor power is transmitted to the worm gear reducer of rolling machine, Rolling machine reducer output shaft and the rolling axis linked to achieve blankets up and down. Sprocket gear on the right side of the output shaft rotate synchronously with the rolling axis, through the first stage and second stage chain drive shaft to transmit power to the Axis III, The Axis III is connected to intermediate blanket, So that the intermediate blanket can automatic up and down. In terms of folding function, When the machine starts rolling and climbing the seamless device automatically open, When fully touch the ground the seamless device automatically fold together. In the working process, It maintains a certain angle with the surface of the greenhouse to adjust to greenhouse surface.

\section{The key component design}

\subsection{The seamless device structure design}

The seamless device are designed as shown in Figure 3, The front end of device is associated with the rolling axis, Axis II maintains coaxial with the end of first and second clamping sleeve, the end of first and second clamping sleeve welded hard limit, to avoid collision with the surface of the greenhouse. At the start of climbing, the device automatically open, when fully contacted with the ground, It is folded together.

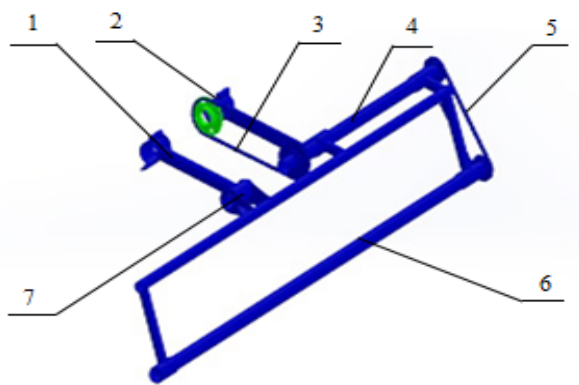

1 First clamping sleeve 2 Second clamping sleeve 3 First-stage transmission chain 4 Axis II 5

Second-stage transmission chain 6 Axis III 7 Hard limit

Figure3 Automatic seamless device 


\subsection{Folding angle of the seamless device}

The folding angle's size depends on the curvature of the surface of greenhouse, It is generally designed with the principle of reasonable axis ${ }^{[4]}$, the formula as follows: $\quad Y=4 F X(L-X) / L^{2}$

In the formula: $\mathrm{Y}$ - Arc height corresponding to each site;

$\mathrm{F}$ - Greenhouse intermediate point height (high vector);

$\mathrm{L}$ - The span of greenhouse;

$\mathrm{X}$ - Members point corresponds to the horizontal distance.

Set the span of greenhouse $\mathrm{L}$ is $8 \mathrm{~m}$, high vector $\mathrm{F}$ is $3.3 \mathrm{~m}$, horizontal distance $\mathrm{X}$ from $1 \mathrm{~m}$ to $4 \mathrm{~m}$, compartment $1 \mathrm{~m}$ takes a value, using MATLAB draw the following curve fitting, shown in Figure 4.

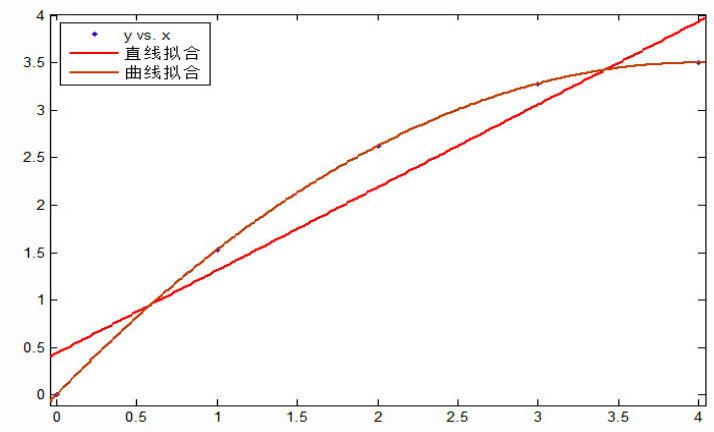

Figure 4 Curve fitting of the solar greenhouse surface

As shown in Figure 4, The curve fitting part is true radians, While the line fitting part is approximate radians $\varphi=\arctan 0.825=38.85^{\circ}$. To ensure the folding function is operating normally, the top of the seamless device neither touch rocker nor touch the surface of greenhouse, the final folding angle is set to $150^{\circ}$.

\section{The control system design}

\subsection{Overall design}

The control system is shown in Figure 5, the entire system consists of a manual switch, remote control module, a mobile phone GSM module, control unit, drive module and position feedback sensors and other components. The control box set up a three-way switch, site manual, remote control and mobile phone messages, The operator not only can use the remote control to operate a plurality of rolling machines, but can simultaneously control the multiple rolling machines. Using a contact switch as a position sensor limits to ensure the safety and reliability of rolling machine.

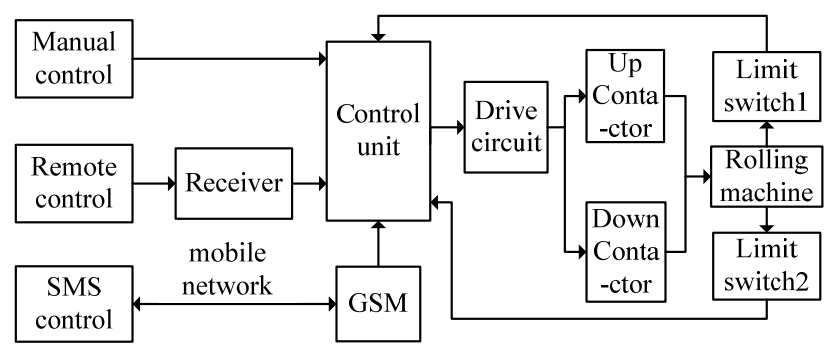

Figure 5 The structure of control system

\subsection{The remote control design}

Remote control system consists of two parts, which is transmitting system and receiving system. The transmitting system is composed of count address circuit, coding circuit, radio transmitter circuit, and the receiving system consists of radio receiver, decoder circuit, switch control circuit.

The radio remote control switch system uses a CMOS integrated circuit, the transmitter module uses PT2262 chip encoding, receiver module with PT2272 chip to decode, while using digital wireless coding technology to ensure the switch without disturbing each other. There are three buttons, which are the up button, down button and stop button. Remote control system has a learning function, By learning to achieve a single controller control multiple rolling machines. 


\subsection{GSM communication module}

GSM module can receive and send messages, Single-chip microcomputer decodes SMS control instructions and completes the appropriate control. The system uses siemens TC35i industrial-grade GSM module, Data interface via AT commands and two-way data transfer instructions, Automatic baud rate is $1.2 \sim 11.5 \mathrm{kB} / \mathrm{s}$, Support for SMS PDU mode message.

When switched off, the SIM card is installed in the SIM card reader,At first, the users must to match their phone to the GSM module, Send text messages to GSM module:your phone number such as 88888888888 , which can control the rolling machine, only the current phone is able to control the rolling machine. Then set the working time, Send messages to GSM module: Time xx seconds or Time xx minutes. At last, Send messages to GSM module:up or down, Achieving SMS remote control.

\section{Test}

To verify the practicality and reliability of solar greenhouse seamless rolling machine, We had tested it in science park of agricultural university of Hebei from 2015.10 to 2016.3. The test results are shown in figure 6.

Tests show that the control system is stable, three kinds of operating mode can be switched freely, the distance of remote control is measured more than 500m and the SMS control function is normal. The intermediate blanket can up and down along with the left and right blankets. The average speed is $1.5 \mathrm{~m} / \mathrm{min}$. It is able to complete a lap in $15 \mathrm{~s}$, It rolled or spread the $10 \mathrm{~m}$ blanket just need $5 \sim 8 \mathrm{~min}$. The folding angle of the seamless device maintains $150^{\circ}$, it adapted to the curvature of the greenhouse surface without collision. In terms of folding, the device can be automatically folded. During the test, the machine is operating normally, safelly and reliable.

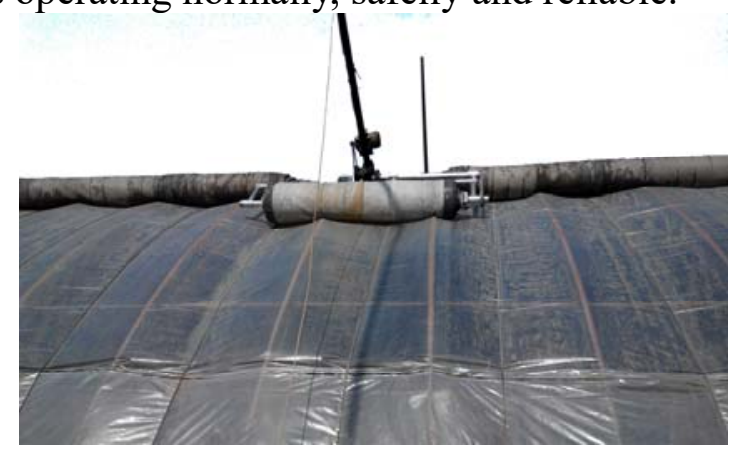

Figure 6 Seamless rolling machine rolling effect

\section{Conclusions}

The seamless rolling machine can automatic roll up and down the intermediate blanket and has a folding function, It greatly reduced farmer's workload and improved the safety of operation. At the start of climbing, the seamless device automatically open $150^{\circ}$ to adapt to the curvature of the greenhouse surface without collision; When fully touch the ground the seamless device automatically fold together. The control system has site manual, short-range remote control and SMS remote control three modes, Using location switch and time protection to achieve double protection. The test result shows that the machine is not only running well but also safe and convenient to operate, The intermediate blanket realizes automatically rolled up and down.

\section{References}

[1] Li Quiguo. Alternate Translations for The Function of Rolling Machine in Facility Agriculture [J] Agricultural Machinery of Hebei,2014.01;pp.101-102.

[2] Ding Xiaoming. Research and Development on Exterior Cover-rolling Machine of Solar Greenhouse in china [J] Journal of Agricultural Mechanization Research, 2011.12;pp.217-222 
[3] Zhang Ying. The Design of Solar Greenhouse and Plastic Greenhouse and Analysis of Construction for Tibet $[\mathrm{J}]$ Science and Technology Mommunication,

2011.02;pp.146-147.

[4] [4] Zhang Ying. The Design of Solar Greenhouse and Plastic Greenhouse and Analysis of Construction for Tibet [J] Science and Technology Mommunication, 2011.02;pp.146-147.

[5] Zhang Yaxin,Cao Wancang. Design of Covered Solar Greenhouse Automatic Control System Based on MCU [J] Chinese Ayicultural Mechanization, 2012.05;

pp.97-100.

[6] Li Duo,Chen Xuedong. Design and Application of Curtain Roiling Machine Automatic Control System for Greenhouse [J] N ingxia Journal of Agriculture and Fores. Sci\& Tech.2012.53(12);pp.181-182.

[7] Wang Jianchun,ChenLiangfu. Application of Automatic Control Technology in Traditional Greenhouse [J] Auhui Agri.Soi.Bull,2015.21(15);pp.57-58. 